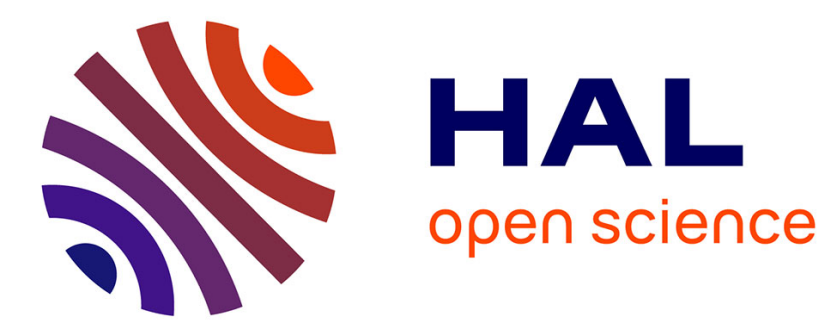

\title{
ProTraS: A probabilistic traversing sampling algorithm
} F. Ros, S. Guillaume

\section{To cite this version:}

F. Ros, S. Guillaume. ProTraS: A probabilistic traversing sampling algorithm. Expert Systems with Applications, 2018, 105, pp.65-76. 10.1016/j.eswa.2018.03.052 . hal-02607456

\section{HAL Id: hal-02607456 https://hal.inrae.fr/hal-02607456}

Submitted on 16 May 2020

HAL is a multi-disciplinary open access archive for the deposit and dissemination of scientific research documents, whether they are published or not. The documents may come from teaching and research institutions in France or abroad, or from public or private research centers.
L'archive ouverte pluridisciplinaire HAL, est destinée au dépôt et à la diffusion de documents scientifiques de niveau recherche, publiés ou non, émanant des établissements d'enseignement et de recherche français ou étrangers, des laboratoires publics ou privés. 


\title{
ProTraS: A Probabilistic Traversing Sampling Algorithm
}

\author{
Frédéric Ros ${ }^{\mathrm{a}, *}$, Serge Guillaume ${ }^{\mathrm{b}}$ \\ ${ }^{a}$ Laboratory PRISME, Orléans university, France \\ ${ }^{b}$ ITAP, Irstea, Montpellier SupAgro, Univ Montpellier, Montpellier, France
}

\begin{abstract}
In the process of knowledge discovery in big data, sampling is a technological brick that can be included in a more general framework to speed up existing algorithms and contribute to the scalability issue. Two challenging and connected problems arise with complexity: tuning and timing. ProTras 1 is a new algorithm that fulfills both requirements. It is driven by a unique parameter, the sampling cost. The cost is overestimated by the maximum within group distance and the group cardinality. It is an iterative algorithm, at each step a new representative is added, chosen as the farthest-first traversal item from the representative in the group with the highest probability of cost reduction. The novel algorithm is robust to noise and time optimized. A detailed comparison with alternative algorithms, conducted on various synthetic and real world data sets, shows that the proposal yields competitive results in terms of quality of representation for clustering, sampling size and sampling time.
\end{abstract}

Keywords: Distortion cost, clustering, distance, density

\section{Introduction}

Defining a sample that behaves like the whole data set is a quite longstanding issue in data management. It has received fresh interest with the

\footnotetext{
* Corresponding author

Email addresses: frederic.ros@univ-orleans.fr (Frédéric Ros), serge.guillaume@irstea.fr (Serge Guillaume)

${ }^{1} \mathrm{~A}$ sample code is available at: http://frederic.rosresearch.free.fr/mydata/ homepage/
}

Preprint submitted to Expert Systems With Applications 
challenge of big data, characterized by an increase in the volume, velocity and variety of the data. From the technical point of view, big data require to clean, analyze, secure and provide a granular access to massive data sets. Scalability is of major concern: the challenge is to propose fast, relevant and user-friendly processing techniques for knowledge discovery in such data. Various concepts and tools, including hardware components, e.g. for parallel processing, have been proposed. Sampling is a technological brick that can be included in a more general framework, such as feature selection or data aggregation, and contribute to the management of big data problems. A fast and easy to tune sampling algorithm could be used when human interaction is required, such as in active learning, or as a preprocessing step for more complex algorithms. Hierarchical clustering is an illustrative example: as it has, in its standard version, a $O\left(n^{2}\right)$ complexity, using a sample size 100 times smaller than the original set leads to a running time divided by 10000 !

The first attempt was the Lloyd algorithm designed in 1957, but only published later (Lloyd, 1982). The goal was to find evenly spaced sets of points in subsets of Euclidean spaces, and partitions of these subsets into well-shaped and uniformly sized convex cells. It is closely related to the $k$-means algorithm, first proposed by Macqueen (1967) and made popular by Hartigan (1975), as both minimize the same objective function, called quantization distortion in signal processing. The main difference is that the Lloyd algorithm uses a Voronoi tessellation.

The Lloyd approach was generalized to any distribution, even with discrete components, by Linde, Buzo and Gray (Linde et al. 1980). Their technique does not involve any differentiation. This vectorial quantization yields an optimal codebook. The $L B G$ algorithm is widely used in signal compression, either image or speech.

Recently, the concept of coreset, more precisely $\varepsilon$-coreset, was proposed (Agarwal et al. 2004). The idea is to quantify the distortion of a given monotonic measure when computed on a sample instead of on the whole set. Extensive research has been carried out to generate such coresets in different frame- 
works (Har-Peled \& Mazumdar, 2004).

Sampling and coresets have been applied to clustering. Clustering is an unsupervised task to organize, summarize and finally understand the data. In the field of big data, clustering algorithms are becoming more and more sophisticated in order to deal with complex data of various shapes and densities (Jain 2010). Two challenging and connected problems arise with complexity: tuning and timing. Uniform sampling is the simplest and quickest way to proceed. Unfortunately, it requires very large sample sets to deal with shape and density variability. Smarter and more powerful algorithms have been proposed. These sampling algorithms, a recent review is available in (Ros \& Guillaume, 2016b), are density or distance based and some of them combine the two notions under specific strategies. Density-based methods can be grouped in two main families for density estimation: space partition (e.g. grids, trees) (Palmer \& Faloutsos, 2000 Ilango \& Mohan, 2010) and local estimation, using neighborhood or kernel functions (Kollios et al., 2003). Both are highly sensitive to parameters, cell definition for grids, bandwidth or neighborhood for local estimators. With an inappropriate setting, these methods are either likely to sample noise or to miss regions of interest. Distance-based clustering algorithms are used for sampling with a number of samples much greater than the number of clusters. The most famous representative of this family is the $k$-means (Hartigan \& Wong, 1979). Its sensitivity to initialization has been exhaustively investigated (Zahra et al. 2015; Arthur \& Vassilvitskii, 2007; Celebi et al., 2013). Single scan approaches have also been proposed such as leader (Ling, 1981) clustering. The results are highly dependent on the distance threshold, even with improved versions (Sarma et al., 2013, Viswanath et al. 2013). Farthest first transversal, fft, algorithms (Rosenkrantz et al. 1977) are of interest as they do not require a distance parameter.

Moreover, improvements in accuracy often conflict with time performance, and response time is of major concern nowadays for data processing algorithms. The increased computational cost limits the application of some of the abovementioned algorithms to small or average size data sets. Several techniques 
have been investigated to address these challenges (Lv et al., 2015, Zhong et al. 2015, Ma et al. 2015, Sarma et al. 2013). The stratification concept has been proposed to speed up algorithms with quadratic or exponential time complexity. To overcome sensitivity to splitting, extensions have been proposed to work with non disjoint partitions, using replication techniques (Machová et al., 2006).

In Feldman et al. (2011), an Efficient Coreset Construction via Adaptive Sampling was proposed, involving density and distance concepts while biasing the random sampling.

Two fft improved algorithms, DIDES (Ros \& Guillaume, 2016b) and DENDIS (Ros \& Guillaume, 2016a), were recently proposed to tackle the still open twofold challenge of tuning and timing. The two of them are iterative algorithms based on the hybridization of distance and density concepts. They differ in the priority given to distance or density, and in the stopping criterion defined accordingly. They benefit from an internal optimization that makes them faster than any competing approach. Their tuning is quite easy as they have only one meaningful and dimensionless parameter, called granularity. However, they also have limitations. First, the meaning that this unique parameter conveys is not the same for the two proposals, this may be an inconvenient from the user point of view. Second, they use internal heuristics based on parameters inferred from the data. Although the approach has been validated on a wide range of data sets with contrasted characteristics, heuristics elimination is always advisable.

The objective of this study is to propose a new sampling algorithm that is both easy to tune and scalable.

The proposed algorithm is only based on the sampling cost. It is a recursive partitioning algorithm: at each step a new representative is added to the sample until the cost falls below a threshold. The representative is chosen in the group with the highest probability of cost reduction. This probability is computed according to the within group distance and to the representativeness of the sample item. Therefore, ProTraS manages the concepts of distance and density in a new probabilistic way. The representative is the farthest item from the group representative. This ensures space coverage. ProTraS is explicitly 
designed to produce a $(k, \varepsilon)$-coreset: the approximation level, $\varepsilon$, is its unique parameter and it is also used as the stopping criterion.

The paper is organized as follows. The fft algorithms, as well as the recent proposals DIDES and DENDIS, are detailed in Section 2. The relationship to the concept of coreset is analyzed in Section 3. The proposed algorithm is introduced in Section 4 and its properties are illustrated using synthetic data. Section 5 deals with the numerical experiments conducted on twenty-one synthetic and eight real world data sets. The sample representativeness is assessed with two well-known, and representative, algorithms, DBSCAN and k-means. The sensitivity to the unique parameter, the distortion cost, is analyzed and a detailed comparison with selected competitors is carried out. Finally, the main conclusions and open perspectives are stated in Section 6 .

\section{Previous work: fft algorithms}

Sampling (Wang et al., 2011, Thompson, 2012) is an interesting way to ensure process tractability. A recent review shows that many algorithms have been proposed since the first attempt, which was the random sampling (Ros \& Guillaume, 2016b). They are based on density or distance, and sometimes combine the two concepts. Among the distance based sampling method, the farthest-first traversal principle is of special interest as it does not require any distance parameter in contrast to the leader (Ling, 1981) or the mountain (Yager \& Filev, 1994) methods. The first use of fft aimed to solve the traveling salesman problem in 1977 (Rosenkrantz et al., 1977). Then the idea was applied to clustering (Gonzalez, 1985), to minimize the maximum distance from any point to its nearest center, and, later, for image color quantization (Xiang, 1997). To initialize the $k$-means algorithm, $k$-means ++ (Arthur \& Vassilvitskii, 2007) uses a fft-like algorithm: a probabilistic component is added to avoid the selection of outliers.

The fft algorithm iteratively adds a new item to the sample at each loop, until a stopping criterion is met. The new representative is the farthest from 
the already selected ones and the stopping criterion in the original version was the sample size. The concept is summarized in Algorithm 1 .

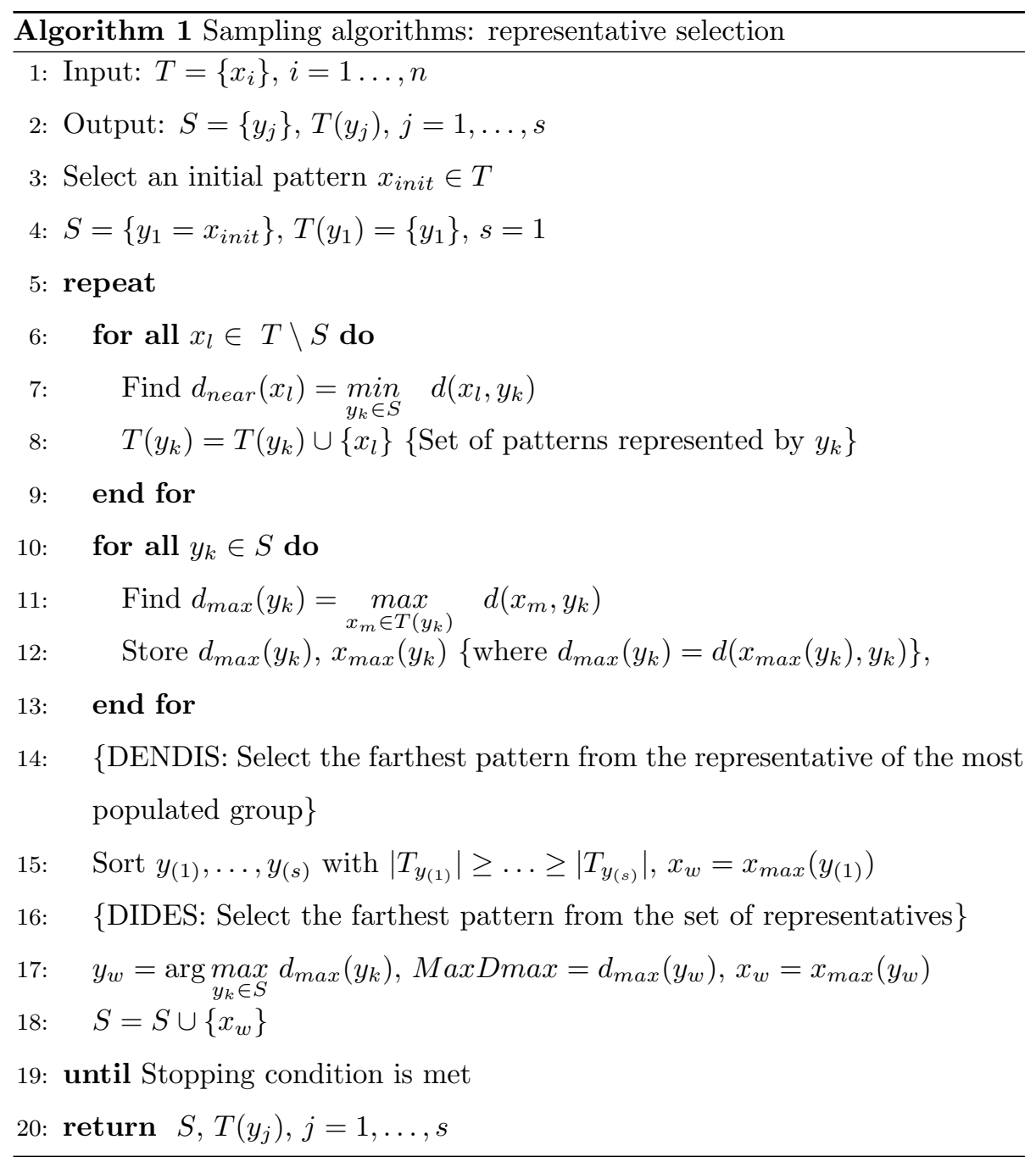

Let $T=\left\{x_{i}\right\}$ be the input set of $n$ multidimensional data, and $S=\left\{y_{j}\right\}$ the size- $s$ sample to be built, $S \subset T$. The set of patterns represented by $y_{k}$ is: $T\left(y_{k}\right)=\left\{x_{i} \mid d\left(x_{i}, y_{k}\right)=\min _{j} d\left(x_{i}, y_{j}\right)\right\}$.

The first pattern can be randomly chosen or it can be computed as the farthest, depending on the selected distance, from the minimum value in each input space dimension. After the initialization phase, the set $S$ only counts this 
initial pattern, $x_{\text {init }}$ (lines 3-4).

The main loop (lines 5-19) includes two steps. First, each unselected pattern, $x \in T \backslash S$, is attached to the closest selected one in $S$ (lines 6-9). At the first step, $T\left(y_{1}\right)=T \backslash\left\{x_{i n i t}\right\}$. Then, for each set $T\left(y_{k}\right)$, the algorithm searches for the farthest attached pattern. The maximum distance in the group represented by $y_{k}$ is noted $d_{\max }\left(y_{k}\right)$ (lines $\left.10-13\right)$.

The next selected representative, $x_{w}$, is the farthest item chosen in a given group. In the pioneering version it was chosen as the farthest of all the already selected items. So, boundary patterns are first chosen instead of inner ones. This way, the selected set spans the whole input space.

Recently, two fft improvements that combine distance and density concepts were proposed. DIDES (Ros \& Guillaume, 2016b) and DENDIS (Ros \& Guillaume, 2016a) differ in the group the new sample item is chosen from and, consequently, in the stopping criterion. The two algorithms are driven by a unique and meaningful parameter, called granularity. It impacts the $S$ size, in that the lower the granularity the higher the number of representatives. It is data independent, and is combined with the whole set cardinality, $n$, to define a threshold: $t h=n$ granularity .

In DENDIS, density is of prime concern while distance is controlled: the representative is chosen in the most populated group. The stopping criterion is also based upon density: the algorithm stops when there are no more groups with a number of attached patterns higher than the threshold and with a maximum distance in the group high enough with respect to the whole distribution.

In DIDES, distance is the dominant criterion and the new representative is the farthest item from the already selected ones, as in the pioneering version. It is chosen in the group which corresponds to the maximum of the $d_{\max }$, MaxDmax (lines 16-17). The threshold th is the minimum size, in the initial set, $T$, for a cluster one wants to have representatives in $S$. A representative with fewer than th patterns attached is called a poor representative. When the proportion of $T$ whose representative is a poor representative is high enough, the input space is homogeneously covered. Then, the $d_{\max }$ evolution curve can 
be modeled to define the stopping criterion as a distance threshold. Density is managed in a post-processing step to discard outliers and consider the representation of connected areas.

The main goal of DIDES is to ensure space coverage while the one of DENDIS is density representation. However, the two algorithms regulate the compromise between these two objectives. This trade-off management requires some specific internal, or hidden, parameters inferred from the data.

\section{Relationship to coresets}

The challenge of big data has aroused a new interest in sampling techniques. But the idea is not new: vector quantization was introduced in the field of signal and image processing to summarize a data distribution by a finite number of vectors (Linde et al. 1980). More recently, the coreset framework introduced the idea of approximation quantification: a subset is called a coreset of a whole set if solving the optimization problem on the subset gives an $\varepsilon$-approximate solution on the whole input set. This section aims to investigate whether there is a relationship between fft algorithms and coresets.

This concept was initially analyzed by Agarwal, Har-Peled and Varadarajan Agarwal et al. 2004) for the geometric approximation of point sets. Given a monotone measure function, $\mu$, i.e. for $S \subseteq T, \mu(S) \leq \mu(T)$, and given $\varepsilon>0$, $S \subseteq T$ is an $\varepsilon$-coreset for $T$ with respect to $\mu$, if $(1-\varepsilon) \mu(T) \leq \mu(S)$. Typical measures include statistics about the set itself such as diameter, width or the geometric shape enclosing $T$, e.g. the smallest enclosing ball characteristics such as radius or volume.

They proved that this approximation can be obtained using a sample whose size is independent of the number of points and only dependent on $\varepsilon$.

This concept has been extended to clustering applications (Har-Peled \& Mazumdar, 2004). The authors proposed the following definition. 
Definition. A set $S$, of $s$ items, is an $(k, \varepsilon)$-coreset for a set $T$, of $n>s$ items if:

$$
(1-\varepsilon) \operatorname{Cost}_{T}(C) \leq \operatorname{Cost}_{S}(C) \leq(1+\varepsilon) \operatorname{Cost}_{T}(C)
$$

where $C=\left\{c_{1}, \ldots, c_{k}\right\}$ is a set of $k$ centers.

Let $c_{i}^{*} \in C$ be the closest center for a given $x_{i} \in T: d\left(x_{i}, c_{i}^{*}\right)=\min _{m \in 1, \ldots, k} d\left(x_{i}, c_{m}\right)$.

Similarly, let $c_{j}^{*^{\prime}} \in C$ be the closest center for a given $y_{j} \in S: d\left(y_{j}, c_{j}^{*^{\prime}}\right)=$ $\min _{m \in 1, \ldots, k} d\left(y_{j}, c_{m}\right)$.

With the $k$-means algorithm, the two costs are:

- $\operatorname{Cost}_{T}(C)=\sum_{i=1}^{n} d\left(x_{i}, c_{i}^{*}\right)$

- $\operatorname{Cost}_{S}(C)=\sum_{j=1}^{s} w_{j} d\left(y_{j}, c_{j}^{*^{\prime}}\right)$, with $w_{j}=\left|T\left(y_{j}\right)\right|$, i.e. the number of items from $T$ whose closest point in $S$ is $y_{j}$.

When this definition only holds for the optimal number of centers, $k, S$ is called a weak coreset for $T$, otherwise, if it holds for any set $C$, it is called a strong coreset for $T$.

Theorem. fft algorithms yield a $(k, \varepsilon)$-coreset.

The proof is as follows:

As $y_{j}$ also belongs to $T$, let $d\left(y_{j}, c_{i}^{*}\right)$ be the distance between the representative and its closest center computed from the whole set $T$.

One obtains $\forall j \in S: d\left(y_{j}, c_{i}^{*}\right)>d\left(y_{j}, c_{j}^{*^{\prime}}\right)$ if $c_{i}^{*} \neq c_{j}^{*^{\prime}}$ otherwise $d\left(y_{j}, c_{i}^{*}\right)=$ $d\left(y_{j}, c_{j}^{*^{\prime}}\right)$ then:

$$
\sum_{j=1}^{s} w_{j} d\left(y_{j}, c_{j}^{*^{\prime}}\right) \leq \sum_{j=1}^{s} w_{j} d\left(y_{j}, c_{i}^{*}\right)
$$

The triangle inequality yields: $d\left(y_{j}, c_{i}^{*}\right) \leq d\left(x, y_{j}\right)+d\left(x, c_{i}^{*}\right)$

The maximum within group distance, $d_{\max }\left(y_{j}\right)=d_{j}$ for group $j$, provides an easy to estimate upper bound of any elementary distance to the center. For 
each group $j \in S$, the inequality can be applied to the set of all the items in the group:

$$
w_{j} d\left(y_{j}, c_{i}^{*}\right) \leq w_{j} d_{j}+\sum_{l=1}^{w_{j}} d\left(x_{l}, c_{i}^{*}\right)
$$

Considering the whole set of the representatives the index of the summation covers the $n$ items and combining Eq 22 and Eq. 3 yields: $\sum_{j=1}^{s} w_{j} d\left(y_{j}, c_{j}^{*^{\prime}}\right) \leq$ $\sum_{j=1}^{s} w_{j} d_{j}+\sum_{i=1}^{n} d\left(x_{i}, c_{i}^{*}\right)$. Finally:

$$
\operatorname{Cost}_{S}(C) \leq \sum_{j=1}^{s} w_{j} d_{j}+\operatorname{Cost}_{T}(C)
$$

The quantity $\sum_{j=1}^{s} w_{j} d_{j}$ is the sampling cost, or the quantization distortion.

The triangle inequality also gives: $d\left(x, c_{j}^{*^{\prime}}\right) \leq d\left(x, y_{j}\right)+d\left(y_{j}, c_{j}^{*^{\prime}}\right)$

then $d\left(y_{j}, c_{j}^{*^{\prime}}\right) \geq d\left(x, c_{j}^{*^{\prime}}\right)-d\left(x, y_{j}\right)$

A similar reasoning, which comes to apply the latter first to a group using $d_{j}$ as an upper estimate of the distance, second to all the groups, and finally stating that $\forall x, d\left(x, c_{j}^{*^{\prime}} \geq d\left(x, c_{i}^{*}\right)\right.$, gives the following inequality:

$\sum_{j=1}^{s} w_{j} d\left(y_{j}, c_{j}^{*^{\prime}}\right) \geq \sum_{i=1}^{n} d\left(x_{i}, c_{i}^{*}\right)-\sum_{j=1}^{s} w_{j} d_{j}$

Meaning:

$$
\operatorname{Cost}_{S}(C) \geq \operatorname{Cost}_{T}(C)-\sum_{j=1}^{s} w_{j} d_{j}
$$

The final relation between the two costs is:

$$
\operatorname{Cost}_{T}(C)-\sum_{j=1}^{s} w_{j} d_{j} \leq \operatorname{Cost}_{S}(C) \leq \operatorname{Cost}_{T}(C)+\sum_{j=1}^{s} w_{j} d_{j}
$$

Dividing Eq. 5 by $\operatorname{Cost}_{T}(C)$, and then multiplying by $\operatorname{Cost}_{T}(C)$, yields:

$$
\begin{aligned}
& \left(1-\frac{\sum_{j=1}^{s} w_{j} d_{j}}{\operatorname{Cost}_{T}(C)}\right) \operatorname{Cost}_{T}(C) \leq \operatorname{Cost}_{S}(C) \leq\left(1+\frac{\sum_{j=1}^{s} w_{j} d_{j}}{\operatorname{Cost}_{T}(C)}\right) \operatorname{Cost}_{T}(C) \\
& \text { which is Eq. } \square 1 \text { with } \varepsilon=\frac{\sum_{j=1}^{s} w_{j} d_{j}}{\operatorname{Cost}_{T}(C)} .
\end{aligned}
$$


$S$ is thus a $(k, \varepsilon)$-coreset for $T$, with $\varepsilon$ equals the ratio of the sampling cost to the whole cost. As there is no assumption about $C, S$ is a strong coreset for $T$.

As mentioned above DIDES and DENDIS are driven by a unique parameter called granularity. The sampling cost and, consequently, $\varepsilon$ has a monotonic evolution with respect to this parameter. A lower granularity tends to yield a higher sample size and adding a new item to $S$ decreases both $w$ and $d$.

\section{ProTraS: the proposed algorithm}

The fft-based algorithms yield coresets even if they were not designed with this goal in mind. The objective of the present proposal is to generate coresets.

\subsection{Description of the algorithm}

The approximation level, which is the sampling cost as shown in the previous section, plays a central role in coreset generation. $\varepsilon$, is the unique parameter. It also serves as the stopping criterion: the algorithm ends when the cost is below the threshold. Finally it is used to guide the sampling process itself: at each iteration a new representative is added to the sample, in the group with the highest probability of cost reduction. This tends to limit the sample size.

The algorithm is summarized in Figure 1 .

1. Add a new sample in the group with the highest probability of cost reduction

2. Assign each pattern to the nearest sample

3. Compute Cost

4. If (Cost $>$ CostParam) goto Step 1

Figure 1: ProTraS: summary of the algorithm

The representative is the farthest-first traversal item of a given group.

As the sampling cost in a given group, $j$, is proportional to both the number of attached patterns, $w_{j}=\left|T_{y_{j}}\right|$, and the maximum within group distance, 
$d_{j}=d_{\max }\left(y_{j}\right)$, the new representative is chosen in the group that is likely to best contribute to the global cost, $\varepsilon$, reduction. The probability of cost reduction is a combination of two basic probabilities: the first one according to the distance and the other one according to the number of attached patterns. Each of them is estimated by normalizing the values by the maximum over all the groups.

For a given group, $j$, the probabilities are:

- Density-based probability: $P_{\text {dens }}(j)=\frac{w_{j}}{m_{i} a x} w_{i}$

- Distance-based probability: $P_{d i s t}(j)=\frac{d_{j}}{m_{i} d_{i}}$

Both have the same meaning: the higher the value, the higher the expected cost reduction. The probability of cost reduction is computed as the following combination: $P_{\text {CostRed }}(j)=\frac{w_{j} d_{j}}{\max _{i}\left(w_{i} d_{i}\right)}$.

This probabilistic approach differs from greedy algorithms. For instance in Decision Trees, the node to be split is the one with the highest gain. This requires all the nodes to be tested, i.e. all the gains have to be computed. The probability estimation tends to speed up the sampling process.

The algorithm is detailed in Algorithm 2 .

The initial pattern selection (line 3 ) is no longer done at random. The first representative is the closest to a virtual item computed as the minimum in each dimension. This makes the algorithm fully deterministic.

The main loop, lines 5-23, includes two for loops. The first one is described in Algorithm 1. The second loop is enriched compared to the previous version. It also computes the combined probability according to $\left|T_{y_{k}}\right|$ and $d_{\max }\left(y_{k}\right)$ (line 14), identifies the group with the highest cost reduction probability (line 16) and updates the global cost before splitting (line 18). The new representative is the farthest-first traversal, $x_{*}$, of the group with the highest probability, represented by $y_{*}$. To update the current cost, the cost due to former $y_{*}$ is subtracted and the new costs, induced by $y_{*}$ and $x_{*}$, are added. The cost is computed at each iteration without any additional computational effort. 


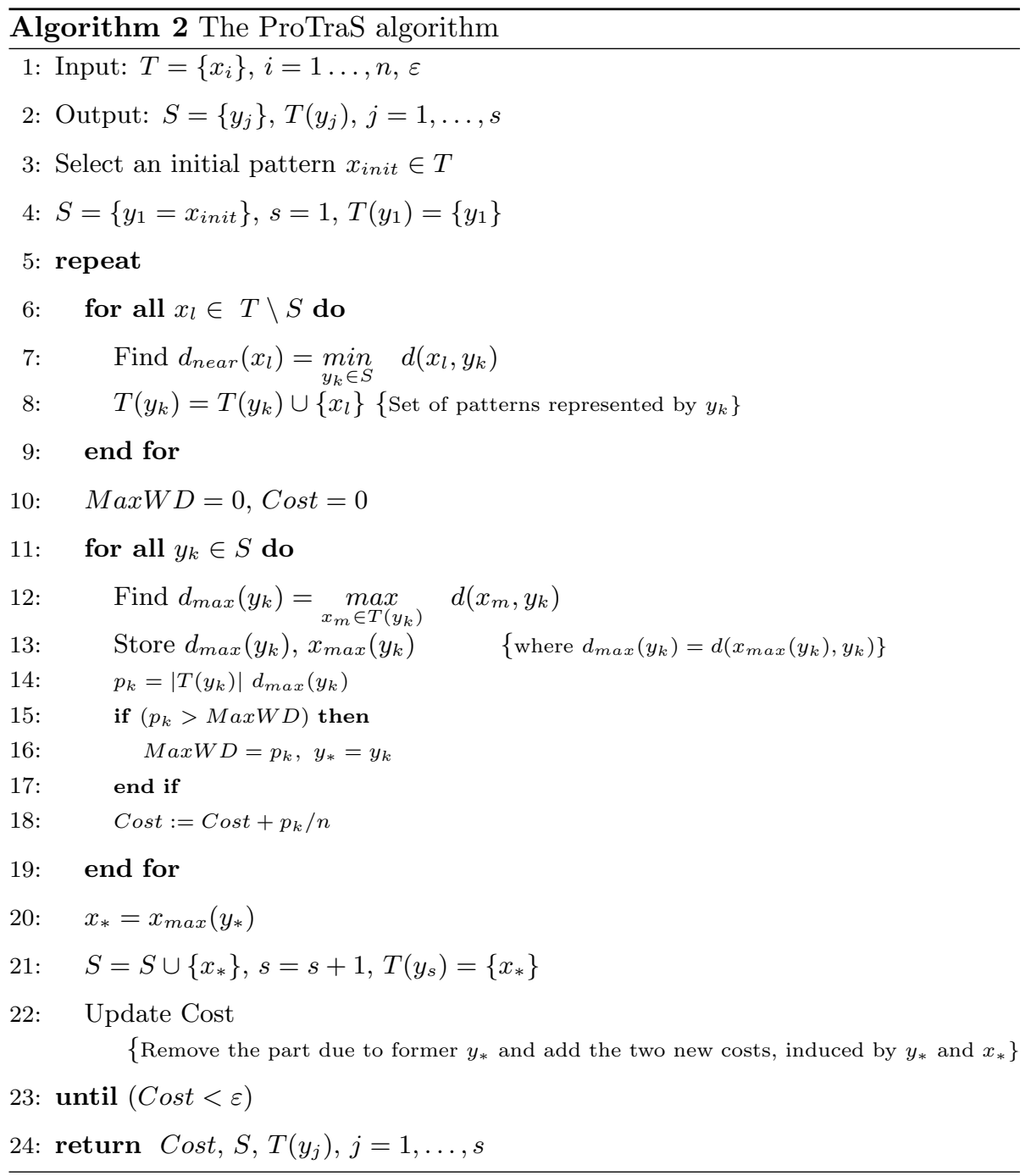


The algorithm uses, in line 18, a normalized distortion cost: $\frac{\sum_{j=1}^{s} w_{j} d_{j}}{n}$, meaning that the approximation, $\varepsilon$, is now a proportion of the whole normalized cost, $\frac{\operatorname{Cost}_{T}(C)}{n}$. This new expression carries the same meaning for all the data, irrespective of the data size.

A better cost estimation could be reached by choosing in each group the medoid as the representative.

\subsection{Algorithm properties}

Some interesting properties of the algorithm are illustrated using the $S 1$ data shown in Figure 2, This synthetic data set includes 3000 2D-points and was used in (Kärkkäinen \& Fränti, 2002) under the name A.set 1.

\subsubsection{Robust to noise}

An increasing amount of uniform random noise (from 1\% to 19\%) was added to the data. The new values were computed independently in each dimension. according to the whole range of the given feature: noise $_{f}=\min _{f}+U[0 .+1] *$ $\left(\max _{f}-\min _{f}\right)$.

To assess the quality of representation, the resulting partitions of the same clustering algorithm applied to the whole set and the selected sample were compared using the Rand Index (Rand, 1971).

As the optimum number of clusters is unknown, tests were carried out within a range $[2,17]$. The $k$-means algorithm was run 10 times for each configuration. Table 1 reports the sample size and the $R I$ averaged over all the trials.

Noise clearly impacts the sampling: the sample size tends to increase with the amount of noise, even if these results also include an initialization variation. $R I$ values show that the sampling algorithm is still able to identify and represent the data structure even with a significant level of noise.

\subsubsection{Sample size mainly dependent on data structure}

The test consists in comparing the algorithm performances on the initial set and an enriched similar one. The latter results from the aggregation of new pat- 
Author-produced version of the article published in Expert Systems with Applications, 2018, №105, p. 65-76.

The original publication is available at https://www.sciencedirect.com

Doi: 10.1016/j.eswa.2018.03.052

Table 1: Noise sensitivity: sample size and $R I$, on $S 1$ data, cost $=0.1$

\begin{tabular}{|c|c|c|c|}
\hline$\%$ noise & Size & $\mathrm{S}$ & $R I$ \\
\hline 0 & 3000 & 261 & 0.975 \\
\hline 1 & 3030 & 309 & 0.979 \\
\hline 2 & 3060 & 323 & 0.978 \\
\hline 3 & 3090 & 342 & 0.990 \\
\hline 4 & 3120 & 348 & 0.984 \\
\hline 5 & 3150 & 362 & 0.982 \\
\hline 6 & 3180 & 356 & 0.977 \\
\hline 7 & 3210 & 364 & 0.978 \\
\hline 8 & 3240 & 369 & 0.978 \\
\hline 9 & 3270 & 368 & 0.985 \\
\hline 10 & 3300 & 378 & 0.983 \\
\hline 11 & 3330 & 374 & 0.984 \\
\hline 12 & 3360 & 370 & 0.975 \\
\hline 13 & 3390 & 384 & 0.988 \\
\hline 14 & 3420 & 381 & 0.977 \\
\hline 15 & 3450 & 380 & 0.976 \\
\hline 16 & 3480 & 392 & 0.985 \\
\hline 17 & 3510 & 388 & 0.983 \\
\hline 18 & 3540 & 388 & 0.978 \\
\hline 19 & 3570 & 393 & 0.979 \\
\hline$\mu$ & & 361 & 0.980 \\
\hline$\sigma$ & & 32 & 0.004 \\
\hline
\end{tabular}




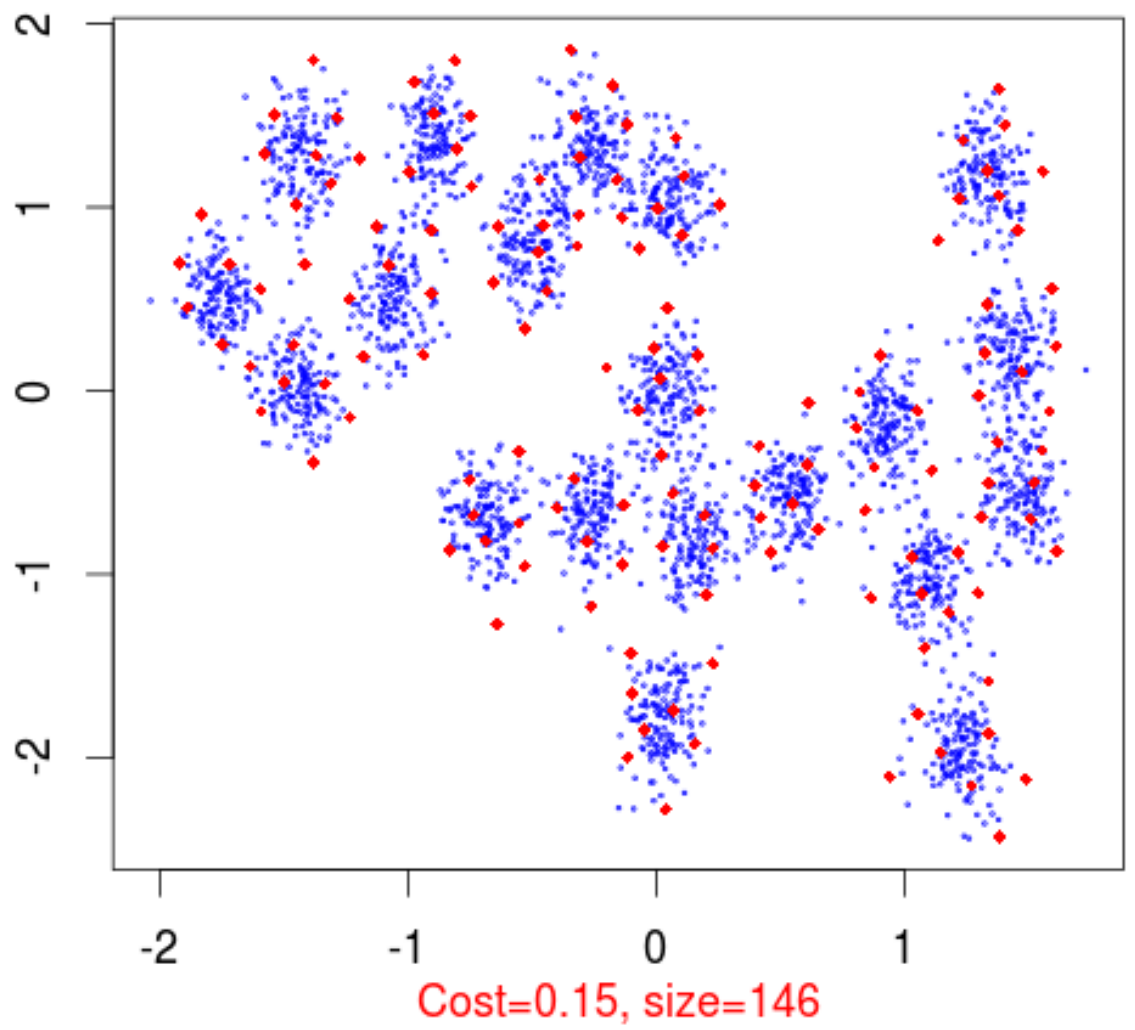

Figure 2: The $S 1$ data

terns: each data point was replicated $k$ times, $k=2, \ldots 20$, with an additional uniform random noise in the range $[-0.1,+0.1] \sigma_{f}$ where $\sigma_{f}$ is the standard deviation for feature $f$. It is worth mentioning that the noise generation is distinct from the one used in the previous section. The expected magnitude is lower as the goal is just to generate non identical items. The sampling is applied with the same cost. In Table 2 the sample size and the $R I$ are given for the initial set, in the first row, and the enriched ones.

The sample size is significantly increased at the first step of the procedure due to the additional noise: the data structure is significantly modified as the noise 
Author-produced version of the article published in Expert Systems with Applications, 2018, №105, p. 65-76.

The original publication is available at https://www.sciencedirect.com

Doi: 10.1016/j.eswa.2018.03.052

Table 2: Data size sensitivity: sample size and $R I$, on $S 1$ data, cost $=0.1$

\begin{tabular}{|c|cc|}
\hline Size & $\mathrm{s}$ & $\mathrm{RI}$ \\
\hline 3000 & 261 & 0.976 \\
6000 & 309 & 0.973 \\
9000 & 323 & 0.980 \\
12000 & 342 & 0.977 \\
15000 & 348 & 0.983 \\
18000 & 354 & 0.970 \\
21000 & 363 & 0.980 \\
24000 & 357 & 0.981 \\
27000 & 366 & 0.979 \\
30000 & 369 & 0.980 \\
33000 & 368 & 0.983 \\
36000 & 377 & 0.984 \\
39000 & 374 & 0.982 \\
42000 & 381 & 0.983 \\
45000 & 376 & 0.983 \\
48000 & 382 & 0.985 \\
51000 & 381 & 0.977 \\
54000 & 380 & 0.976 \\
57000 & 392 & 0.985 \\
60000 & 388 & 0.986 \\
\hline$\mu$ & & 0.980 \\
$\sigma$ & & 0.004 \\
\hline
\end{tabular}


is randomly defined for each input dimension. Then it tends to become more stable. The sample size ranges from 261 to 392 while the whole size becomes 20 times bigger. This does not significantly impact the results. This experiment shows that the outcome of ProTraS depends more on the data structure than on the data size.

\subsubsection{Time optimized}

At a given iteration only a small part of the input space is modified. It is possible to avoid a lot of distance computations using the triangle inequality and a limited amount of memory space.

When a new representative in $S$ has been selected, $y_{*}$, the question is: should a given initial pattern, $x$, be attached to $y_{*}$ instead of remaining in $T\left(y_{j}\right)$ ? In the case illustrated in Figure 3 , three groups are defined and the new representative belongs to the first one. The triangular inequality states: $d\left(y_{j}, y_{*}\right) \leq d\left(x, y_{j}\right)+$ $d\left(x, y_{*}\right)$ and $x$ would be attached to $y_{*}$ if $d\left(x, y_{*}\right)<d\left(x, y_{j}\right)$. So, if $d\left(y_{j}, y_{*}\right) \geq$ $2 d\left(x, y_{j}\right), x$ remains in $T\left(y_{j}\right)$. Storing the maximum within group distance, $d_{\max }\left(y_{j}\right)$, makes the test useless for all the items in the group if the inequality holds for the farthest from $y_{j}$. That means that if $d\left(y_{j}, y_{*}\right) \geq 2 d_{\max }\left(y_{j}\right)$ no change has to be made. This is the case for Group 2 in the upper part of the plot, the farthest item from $y_{2}$ is $x_{2}$.

The number of patterns managed by this group level optimization increases with the sample size, as the averaged induced volume decreases. When the whole group is not managed by the previous test, only one alternative group must be considered, the closest from the one with the new representative, located at the distance $d_{1 n n}(y)$. The same triangle inequality applied at the pattern level provides a useful threshold. All $x \in T\left(y_{j}\right)$ with $d_{\text {near }}(x) \leq 0.5 d\left(y_{j}, y_{*}\right)$ remain attached to $T\left(y_{j}\right) . d_{\text {near }}(x)$ is the distance from $x$ to the closest element in $S$, its representative, see Algorithm [1. In the figure, $y_{*}=y_{4}$ is the farthest item in Group 1, $x_{1}$, and the closest representative from $y_{1}$ is $y_{3}$. The limits of the new group, Group 4, are plotted in dashed lines. Group 3 is reduced and $x_{i}$ becomes the farthest element from $y_{3}$, and it is now labeled as $x_{3}$. Similarly, $x_{j}$ 


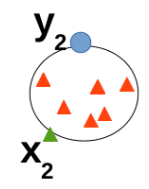

\section{Group 2: $\mathrm{d}\left(\mathrm{y}_{2}, \mathrm{y}_{*}\right)>2 \mathrm{~d}\left(\mathrm{x}_{2}, \mathrm{y}_{2}\right)$ \\ Group 3: $\forall x>x_{i}, d\left(x, y_{3}\right)<.5 d\left(y_{3}, y_{*}\right)$}
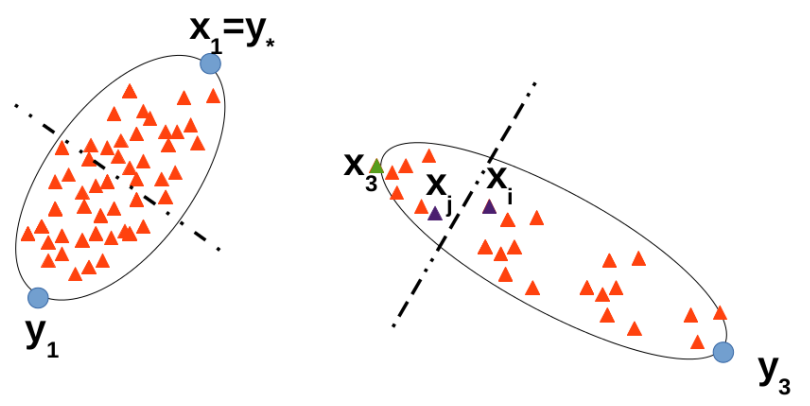

Figure 3: Optimization illustration

becomes $x_{4}$.

The spatial complexity for this time optimization can be considered as reasonable: the final version stores $n+2 * s$ distances. $n d_{n e a r}(x), s d_{\max }(y)$ and $d_{1 n n}(y)$ as well as the corresponding elements, $y$ for $d_{\text {near }}(x)$, and $x$ for $d_{\text {max }}(y)$.

\section{Numerical experiments}

Twenty-nine databases were used, 21 are synthetic, $S 1$ to $S 21$, and 8 are real world data sets, $R 1$ to $R 8$.

The selected data are from the data clustering repository of the computing school of Eastern Finland University ${ }^{2}$, Finland hereafter, the UCI machine learning repository ${ }^{3} U C I$, the github clustering benchmark 4 clustering, or were proposed in the published literature.

\footnotetext{
${ }^{2}$ https://cs.joensuu.fi/sipu/datasets/

${ }^{3}$ https://archive.ics.uci.edu/ml/

${ }^{4}$ https://github.com/deric/clustering-benchmark
} 
Data are of various dimensions (from 2 to 10), sizes (from tiny to very large), shapes and densities. Their main characteristics and origin are summarized in Table 3

The data were standardized to have a zero mean and a unit variance. Some synthetic data sets are plotted in Figure 4

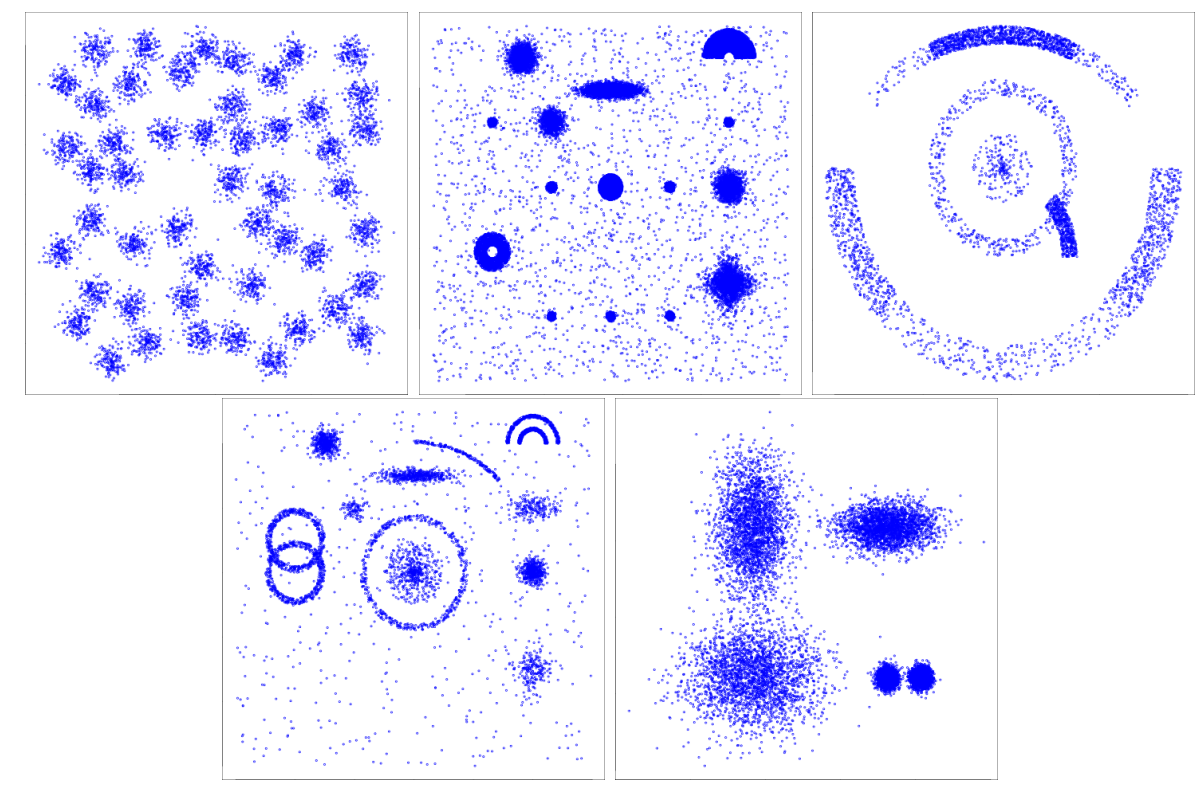

Figure 4: Data sets S3, S17, S18, S20 and S21

\subsection{Sample representativeness}

Two different clustering techniques were tested to assess the sample representativeness: k-means and DBSCAN (Zhou et al. 2000). The former is distance-based, the latter is density-based. Various improvements have been recently proposed (Tran et al., 2013) but they are beyond the scope of this paper. The native versions were used in the present study.

\footnotetext{
${ }^{5}$ The original data set, with 240 items, was enriched up to 12500 items. Each duplicated point is added a random Gaussian noise in each dimension, $f,: x_{f}^{\prime}=x_{f}+\mathcal{N}\left(x_{f}, 0.1 \sigma_{f}\right)$.
} 
Table 3: The synthetic and real world databases

\begin{tabular}{|c|c|c|c|c|c|}
\hline & Size & Dim & Name & \multicolumn{2}{|l|}{ Origin } \\
\hline S1 & 3000 & 2 & A.set 1 & (Kärkkäinen \& Fränti & $\mathrm{i}, 2002)$ \\
\hline $\mathrm{S} 2$ & 5250 & 2 & A.set 2 & (Kärkkäinen \& Fränti & $\mathrm{i},(2002)$ \\
\hline S3 & 7500 & 2 & A.set 3 & (Kärkkäinen \& Fränti & $\mathrm{i},(2002)$ \\
\hline $\mathrm{S} 4$ & $12000^{5}$ & 2 & FLAME & (Fu \& Medico, 2007) & \\
\hline S5 & 100000 & 2 & Birch-set 3 & (Zhang et al., 1997) & \\
\hline S6 & 373 & 2 & JAIN & (Jain \& Law, 2005) & \\
\hline S7 & 5000 & 2 & S.sets 1 & (Fränti \& Virmajoki & 2006) \\
\hline S8 & 5000 & 2 & S.sets 2 & (Fränti \& Virmajoki & 2006) \\
\hline S9 & 5000 & 2 & S.sets 3 & (Fränti \& Virmajoki & 2006) \\
\hline S10 & 5000 & 2 & S.sets 4 & (Fränti \& Virmajoki & 2006) \\
\hline S11 & 1351 & 2 & Dim sets 1 & Finland & \\
\hline S12 & 2701 & 4 & Dim sets 2 & Finland & \\
\hline $\mathrm{S} 13$ & 4051 & 6 & Dim sets 3 & Finland & \\
\hline S14 & 5401 & 8 & Dim sets 4 & Finland & \\
\hline S15 & 6751 & 10 & Dim sets 5 & Finland & \\
\hline S16 & 8000 & 2 & cluto-t8-8k & clustering & \\
\hline S17 & 40000 & 2 & Homemade & Fig. 4 & \\
\hline S18 & 3800 & 2 & Homemade & Fig. 4 & \\
\hline S19 & 8000 & 2 & $\mathrm{t} 4.8 \mathrm{k}$ & (Karypis et al. 1999) & \\
\hline $\mathrm{S} 20$ & 5500 & 2 & Homemade & Fig. 4 & \\
\hline $\mathrm{S} 21$ & 12500 & 2 & Homemade & Fig. 4 & \\
\hline $\mathrm{R} 1$ & 68040 & 9 & Color moments & Finland & \\
\hline $\mathrm{R} 2$ & 169308 & 3 & Differential coordinates & (Fränti \& Virmajoki, & 2006) \\
\hline $\mathrm{R} 3$ & 13467 & 2 & User Location (Finland) & (Fränti \& Virmajoki, & 2006) \\
\hline $\mathrm{R} 4$ & 950 & 10 & Stock & (Alcalá et al., 2010) & \\
\hline R5 & 857357 & 3 & Transactions90k & (Alcalá et al. , 2010) & \\
\hline $\mathrm{R} 6$ & 1837 & 3 & House 5 & (Fränti \& Virmajoki & 2006) \\
\hline $\mathrm{R} 7$ & 34112 & 3 & House8 & (Fränti \& Virmajoki & 2006) \\
\hline $\mathrm{R} 8$ & 45781 & 3 & Tamildanu & $U C I$ & \\
\hline
\end{tabular}


As the optimum number of clusters is unknown, tests were carried out within a range $[2,17]$. Finding one configuration with a given number of clusters with $D B S C A N$ is quite tricky, unlike with $k$-means, for which this number is an input parameter. Using the $k$-means, when the number of clusters gets high, some of them may be very small, or even empty, for a given data set. In this case the range is restricted to the representative clusters.

$D B S C A N$ is run with two parameters: the distance, $d$, that defines a spherical neighborhood, and the minimum number of points, minp, required to consider the data point as a core point, otherwise it is labeled as an outlier. A specific process was designed to set these two parameters in order to obtain a desirable number of clusters with a reasonable number of outliers, less than $10 \%$.

First, $D B S C A N$ was run on the sample with $\min p=\max (1,0.01 n)$ to identify some distances that fit the above mentioned conditions. Let $d_{\text {init }}$ be the distance that gives 2 clusters. Then the range $\left[0, d_{i n i t}\right]$ is equally divided into 1000. DBSCAN was run with all these distance parameters on the samples and on the whole sets. All the configurations that gave an acceptable number of clusters and with the proportion of outliers less than the threshold were kept. The partitions were compared for all the pairs, one element with the sample and the other with the whole set, for which the difference in the number of clusters was at most 2 .

As $D B S C A N$ is computationally expensive, data size was limited to 5000 to complete the test within a reasonable time. The extra items were randomly removed.

As $k$-means is sensitive to initialization, a given number of trials, 10 in this paper, were run for each number of clusters.

The resulting sample size as well as the computational cost were carefully studied as they have a strong impact on the practical use of the algorithm. The CPU cost is characterized by a time ratio. It is computed as the sum of the sampling time and the time for clustering the sample divided by the time required to cluster the whole data. 
As in the previous section, the $R I$ is used to assess the quality of representation. The value reported in the following tables is the average of all experiments, number of runs and number of clusters.

The same cost, 0.1, was used for the sampling. As it is normalized by the data size, it carries the same meaning for all the data sets.

The results are summarized in Table 4 is the whole set size, $s$ the sample one, $s / t$ is thus the sampling ratio, and the $R I$ and time ratio are reported for both algorithms.

It is interesting to see that the sampling ratio ranges from $0 . \%(R 3)$ to $73 \%$ (R4). Similar values are obtained with the synthetic data: $0.4 \%$ for $S 5$ and $70 \%$ for $S 4$. This highlights that there is no relationship between the cost, which is 0.1 for all the data sets, and the sample size or the sampling ratio. This is a strong asset of ProTraS: the sample size depends only on the data structure. The sample size is limited to 1999 by the program. When this limit is reached, the real cost may be higher than the program parameter. This occurred for $R 1$, $R 5$ and $R 8$. Even if the cost is not reached, the representativeness of the sample is satisfactory.

For a large number of clusters, i.e. when $k$ tends to $n$, the relationship between the cost and the $R I$ is clear. With the range studied here, however, it is not so clear. Even if the $R I$ are usually high, some comments are in order. With the $R 2$ data, this value is below 0.9 for the $k$-means. In these cases $D B S C A N$ yields a better index. A detailed analysis of $R 2$ experiments shows that some configurations are not stable for the $k$-means, given this cost $(0.1)$ and the corresponding sample rate (about $1 \%$ ). With $k=2$, the mean $R I$ is 0.76 , and it is 0.77 with $k=5$.

The last two columns of Table 4 report the time ratio given in percent. It is, as expected, clearly lower for $D B S C A N$ than $k$-means. For the latter, sampling becomes interesting when dealing with large databases or well structured data. In this case, the sample size is much smaller than the whole set. 
Table 4: ProTraS with cost $=0.1$ and two clustering algorithms

\begin{tabular}{|c|c|c|c|c|c|c|c|}
\hline & & & & & \{ & Time & atio $(\%)$ \\
\hline & $t$ & $s$ & $s / t$ & k-means & DBSCAN & k-means & DBSCAN \\
\hline S1 & 3000 & 261 & 0.089 & 0.978 & 0.960 & 122.8 & 1.8 \\
\hline $\mathrm{S} 2$ & 5250 & 315 & 0.060 & 0.978 & 0.960 & 52.5 & 0.95 \\
\hline S3 & 7500 & 341 & 0.046 & 0.972 & 0.975 & 45.1 & 0.6 \\
\hline $\mathrm{S} 4$ & 12000 & 133 & 0.696 & 0.952 & 1.000 & 22.5 & 0.29 \\
\hline S5 & 100000 & 259 & 0.004 & 0.979 & 1.000 & 25.7 & 0.03 \\
\hline S6 & 373 & 108 & 0.308 & 0.958 & 0.980 & 178.9 & 18.0 \\
\hline S7 & 5000 & 237 & 0.048 & 0.985 & 1.000 & 52.7 & 0.8 \\
\hline $\mathrm{S} 8$ & 5000 & 327 & 0.066 & 0.981 & 1.000 & 50.0 & 1.1 \\
\hline S9 & 5000 & 422 & 0.084 & 0.981 & 1.000 & 54.8 & 1.3 \\
\hline S10 & 5000 & 448 & 0.084 & 0.969 & 1.000 & 56.7 & 1.3 \\
\hline $\mathrm{S} 11$ & 1351 & 17 & 0.012 & 0.978 & 1.000 & 71.4 & 0.6 \\
\hline $\mathrm{S} 12$ & 2701 & 17 & 0.007 & 0.997 & 0.980 & 30.6 & 0.6 \\
\hline $\mathrm{S} 13$ & 4051 & 20 & 0.106 & 0.995 & 1.000 & 53.8 & 3.4 \\
\hline S14 & 5401 & 416 & 0.277 & 0.994 & 0.990 & 152.2 & 8.8 \\
\hline S15 & 6751 & 379 & 0.296 & 0.995 & 1.000 & 208.6 & 9.9 \\
\hline S16 & 8000 & 303 & 0.061 & 0.993 & 0.980 & 42.3 & 0.08 \\
\hline S17 & 40000 & 475 & 0.006 & 0.976 & 0.940 & 22.3 & 1.0 \\
\hline S18 & 3800 & 236 & 0.044 & 0.979 & 1.000 & 38.8 & 0.7 \\
\hline S19 & 8000 & 475 & 0.060 & 0.971 & 0.980 & 36.8 & 0.1 \\
\hline $\mathrm{S} 20$ & 5500 & 253 & 0.060 & 0.971 & 0.980 & 36.8 & 0.1 \\
\hline $\mathrm{S} 21$ & 12500 & 238 & 0.060 & 0.975 & 0.980 & 36.8 & 0.1 \\
\hline $\mathrm{R} 1$ & 68040 & 1999 & 0.029 & 0.933 & 1.000 & 98.2 & 0.02 \\
\hline $\mathrm{R} 2$ & 169308 & 1008 & 0.011 & 0.884 & 1.000 & 39.1 & 0.03 \\
\hline $\mathrm{R} 3$ & 13467 & 103 & 0.008 & 0.934 & 1.000 & 27.1 & 0.2 \\
\hline $\mathrm{R} 4$ & 950 & 695 & 0.733 & 0.999 & 1.000 & 210.2 & 60.9 \\
\hline $\mathrm{R} 5$ & 857357 & 1999 & 0.020 & 0.991 & 0.985 & 70.2 & 0.004 \\
\hline $\mathrm{R} 6$ & 1837 & 1252 & 0.682 & 0.977 & 1.000 & 312.1 & 51.6 \\
\hline $\mathrm{R} 7$ & 34112 & 1672 & 0.049 & 0.982 & 0.970 & 95.5 & 0.3 \\
\hline $\mathrm{R} 8$ & 45781 & 1999 & 0.027 & 0.961 & 0.950 & 93.1 & 0.05 \\
\hline
\end{tabular}




\subsection{Cost sensitivity}

The cost plays a central role in the ProTraS algorithm. Not only is it the input parameter, it is also used to select the sample items and serves as a stopping criterion. To assess the algorithm sensitivity to the cost, 10 cost values, from 0.08 to 0.26 by steps of 0.02 , were used with all the data sets. For each data set and a given cost the $k$-means was run 10 times with a number of clusters from 2 to 17 on the sample and the whole set. The $R I$ was averaged over all these trials.

The results are summarized in Table 5 The first two columns report the maximum and minimum sample size over all the costs, the following two ones show the same information for the $R I$, and finally the differences are computed for both indicators.

The maximum size was limited to 1999 in 5 cases. In one of them, $R 1$, it is also the minimum size, meaning that none of the cost approximations could be reached with such a limited size. For the $R 1$ data, the differences in the $R I$ are due only to the $k$-means initialization.

For 3 data sets, the difference in the sample size is higher than 1500: $S 14$, $R 7$ and $R 8$. The corresponding variation for the $R I$ is not that high.

The expected trend is that the smaller the cost, the better the representation will be, but the sample size and the $R I$ are also determined by the data structure. Two real data sets, $R 2$ and $R 3$ have a minimum $R I$ less than 0.9 .

These experiments show that for a large range of distortion costs, the sample is able to well represent the whole according to the $R I$ even with a reduced size. This highlights the quality of the representative selection using the ProTraS algorithm.

\subsection{Comparison with alternative approaches}

This section focuses on the comparison between ProTraS, DENDIS, DIDES and two of the twelve methods already used to assess the relevance of DENDIS or DIDES. The two of them proved competitive regarding their computational 
Table 5: Cost sensitivity, with $R I$ for the $k$-means

\begin{tabular}{|c|c|c|c|c|c|c|}
\hline & $s_{\operatorname{Max} C}$ & $s_{M i n C}$ & $R I_{\text {Max } C}$ & $R I_{M i n C}$ & $\Delta s$ & $\Delta R I$ \\
\hline$S 1$ & 353 & 66 & 0.988 & 0.934 & 287 & 0.054 \\
\hline$S 2$ & 429 & 76 & 0.982 & 0.941 & 353 & 0.040 \\
\hline$S 3$ & 472 & 74 & 0.979 & 0.939 & 398 & 0.040 \\
\hline$S 4$ & 403 & 87 & 0.976 & 0.943 & 316 & 0.033 \\
\hline$S 5$ & 360 & 70 & 0.984 & 0.939 & 290 & 0.044 \\
\hline$S 6$ & 140 & 37 & 0.962 & 0.951 & 103 & 0.011 \\
\hline$S 7$ & 332 & 65 & 0.981 & 0.960 & 267 & 0.022 \\
\hline$S 8$ & 438 & 82 & 0.992 & 0.951 & 356 & 0.040 \\
\hline$S 9$ & 582 & 106 & 0.988 & 0.945 & 476 & 0.043 \\
\hline$S 10$ & 582 & 106 & 0.980 & 0.953 & 476 & 0.027 \\
\hline$S 11$ & 25 & 10 & 0.974 & 0.968 & 15 & 0.006 \\
\hline$S 12$ & 41 & 10 & 0.996 & 0.969 & 31 & 0.027 \\
\hline$S 13$ & 721 & 21 & 0.995 & 0.960 & 700 & 0.035 \\
\hline$S 14$ & 1999 & 69 & 0.996 & 0.965 & 1930 & 0.030 \\
\hline$S 15$ & 472 & 164 & 0.998 & 0.987 & 308 & 0.011 \\
\hline$S 16$ & 666 & 102 & 0.976 & 0.935 & 564 & 0.041 \\
\hline$S 18$ & 302 & 92 & 0.991 & 0.974 & 210 & 0.017 \\
\hline$S 19$ & 298 & 53 & 0.972 & 0.934 & 245 & 0.038 \\
\hline$S 20$ & 335 & 80 & 0.990 & 0.952 & 255 & 0.035 \\
\hline$S 21$ & 239 & 47 & 0.982 & 0.945 & 192 & 0.037 \\
\hline$R 1$ & 1999 & 1999 & 0.937 & 0.929 & 0 & 0.008 \\
\hline$R 2$ & 1446 & 214 & 0.930 & 0.889 & 1232 & 0.041 \\
\hline$R 3$ & 130 & 43 & 0.928 & 0.912 & 87 & 0.016 \\
\hline$R 4$ & 736 & 412 & 0.997 & 0.980 & 324 & 0.017 \\
\hline$R 5$ & 1999 & 533 & 0.987 & 0.968 & 1466 & 0.019 \\
\hline$R 6$ & 1369 & 260 & 0.981 & 0.942 & 1109 & 0.040 \\
\hline$R 7$ & 1999 & 237 & 0.983 & 0.953 & 1762 & 0.031 \\
\hline$R 8$ & 1999 & 416 & 0.984 & 0.962 & 1583 & 0.022 \\
\hline
\end{tabular}


cost and quality of representation. To be fair, the comparison is carried out with a same sample size, the one given by the ProTraS algorithm.

The first one, denoted AdaCor, described in (Feldman et al., 2011), proposes an Efficient Coreset Construction via Adaptive Sampling. The key idea is to build an approximate solution (sample set, $S$ ) and to use it to bias the random sampling. The first step is achieved by an iterative algorithm that samples a small number of points, $\beta$, and removes half of the data set $(T)$ closest to the sampled points. In the second step the sampling is biased with probabilities, for each point in $T$, which are roughly proportional to their squared distance to $S$.

The second approach, denoted AdaGri, combines the pioneering idea from Kollios et al. (2003) to bias the sampling process with an adaptive grid partitioning (Lin et al., 1997; Pintore et al. 2002). At each step the densest bin (subspace) is split. The final subspace set is used to estimate the local densities $\hat{f}(x)$.

Once the local densities have been estimated, they are used to bias the sampling process as follows:

$$
\frac{s}{\sum \hat{f}(x)} \hat{f}(x)^{\alpha}
$$

where $s$ is the desired sample size and $\alpha$ the bias parameter. If $\alpha=0$, the process reduces to a random sampling $\left(\frac{s}{n}\right)$. Otherwise, if $\alpha>0$ (respectively $\alpha<0$ ) high density regions are sampled at a higher (lower) rate.

This algorithm is sensitive to its parameters: the bias, $\alpha$, the number of cuts tested per axis, $N_{c u t}$, the maximum number of bins $\operatorname{Max}_{b}$ and the minimum number of points in a bin, $O c c_{m}$.

A given cost from ProTraS yields a sample size which is used as an input for the other algorithms. The relevant input parameters for AdaCor and AdaGri are similar to the ones used by the authors in the referenced papers: $\beta=$ $100, \alpha=-0.15, N_{c u t}=2, \operatorname{Max}_{b}=200, O c c_{m}=5 . D E N D I S$ and DIDES are run with the granularity that yields a similar number of samples to ProTraS. To avoid a random effect, these two algorithms are initialized, as ProTraS, by the $f f t$ from a virtual extreme item. Only AdaCor is thus sensitive to random, 
the three others being deterministic. 10 samples are generated from AdaCor for each configuration. For each data set, the $k$-means was run 10 times with a number of clusters from 2 to 17, both on each of the samples and the whole set. The results were averaged over all these trials. The comparison is made, for the same sample size, on the running time and the quality of representation. As the running time for DENDIS and DIDES is similar to the one of ProTraS, it is not analyzed.

Two contrasted cases were studied, corresponding to two costs: a high approximation level, 0.1 , and a low one, 0.2 . The results are given in Tables 6 and 7.

The high approximation level yielded quite good results with ProTraS: the mean $R I$ for the synthetic data is 0.979 , it is higher than 0.95 in all cases and above 0.99 for 7 sets. For any alternative approach the result shown in the tables is computed as the ProTraS value minus the alternative corresponding one. DENDIS was better than ProTraS for $S 6$ and $S 21$ when an absolute value of 0.005 for the $R I$ was used as a threshold of significance. In average, DENDIS and AdaCor performed better than DIDES and AdaGri with the synthetic data. AdaCor proved slower than the others. For the eight real world data sets, the average quality was decreased to 0.961 for ProTraS and, in two cases, AdaCor and AdaGri did better than the proposal. The standard deviation of the RI are quite similar, ranging between 0.02 in average for ProTraS and DIDES to 0.04 for the others.

The result for the low approximation level are given in Table6. The samples were, as expected, significantly smaller and the running time for ProTraS was half of the other one in average. The quality of representation is also decreased: only two $R I$ for the synthetic data were higher than 0.99 and two of them are below 0.95 . The difference in the averaged $R I$ was increased for all the competitors except DIDES, which yielded a similar result to the previous experiment. With the real data, the result of the low level approximation is confirmed: $A d a-$ Cor gave a similar quality of representation to ProTraS, but it is much slower. The standard deviation of the $R I$ was also decreased by a factor 2 with respect 
Table 6: Alternative approaches: comparison for high level approximation

\begin{tabular}{|c|c|c|c|c|c|c|c|c|c|}
\hline & \multicolumn{3}{|c|}{ ProTraS } & \multirow{2}{*}{$\begin{array}{r}D E N D I S \\
\Delta R I\end{array}$} & \multirow{2}{*}{$\begin{array}{r}D I D E S \\
\Delta R I\end{array}$} & \multicolumn{2}{|c|}{ AdaCor } & \multicolumn{2}{|c|}{ AdaGri } \\
\hline & $\mathrm{S}$ & $\mathrm{t}(\mathrm{ms})$ & $R I$ & & & $\Delta t$ & $\Delta R I$ & $\Delta t$ & $\Delta R I$ \\
\hline $\mathrm{S} 1$ & 261 & 25 & 0.978 & 0.008 & 0.017 & -17 & 0.018 & 7 & 0.032 \\
\hline $\mathrm{S} 2$ & 315 & 31 & 0.978 & 0.006 & 0.017 & -68 & 0.021 & 2 & 0.042 \\
\hline S3 & 341 & 36 & 0.972 & 0.017 & 0.014 & -122 & 0.014 & 1 & 0.035 \\
\hline $\mathrm{S} 4$ & 133 & 46 & 0.952 & 0.005 & 0.019 & -215 & 0.032 & 0 & 0.034 \\
\hline S5 & 259 & 207 & 0.979 & 0.123 & 0.130 & -2392 & 0.012 & -152 & 0.050 \\
\hline S6 & 108 & 11 & 0.959 & -0.005 & 0.054 & 8 & 0.001 & 10 & 0.013 \\
\hline $\mathrm{S} 7$ & 237 & 19 & 0.986 & 0.005 & 0.038 & -62 & 0.011 & -5 & 0.024 \\
\hline $\mathrm{S} 8$ & 327 & 24 & 0.981 & 0.017 & 0.048 & -65 & 0.024 & -2 & 0.031 \\
\hline S9 & 422 & 26 & 0.981 & 0.007 & 0.044 & -68 & 0.014 & -1 & 0.025 \\
\hline S10 & 448 & 36 & 0.969 & -0.001 & 0.016 & -59 & 0.018 & 9 & 0.055 \\
\hline S11 & 17 & 26 & 0.979 & 0.005 & 0.047 & -68 & 0.013 & -1 & 0.027 \\
\hline S12 & 17 & 6 & 0.997 & 0.034 & 0.029 & -6 & 0.070 & -1 & 0.088 \\
\hline S13 & 20 & 9 & 0.995 & 0.038 & 0.012 & -26 & 0.020 & -16 & 0.032 \\
\hline S14 & 476 & 46 & 0.994 & 0.012 & -0.002 & -70 & 0.000 & -26 & 0.012 \\
\hline S15 & 379 & 126 & 0.995 & -0.001 & 0.005 & -143 & -0.001 & 36 & 0.005 \\
\hline S16 & 303 & 221 & 0.993 & -0.004 & 0.020 & -265 & -0.002 & -12 & -0.004 \\
\hline S17 & 475 & 38 & 0.977 & 0.006 & 0.046 & -147 & 0.009 & -9 & 0.045 \\
\hline S18 & 236 & 98 & 0.979 & 0.055 & 0.119 & -1682 & 0.043 & -57 & 0.057 \\
\hline S19 & 475 & 71 & 0.971 & 0.008 & 0.043 & -116 & 0.005 & 23 & 0.023 \\
\hline S20 & 253 & 27 & 0.971 & 0.022 & 0.097 & -68 & 0.010 & -1 & 0.032 \\
\hline S21 & 238 & 39 & 0.975 & -0.008 & 0.090 & -252 & 0.012 & -17 & -0.002 \\
\hline mean & & 55 & 0.979 & 0.017 & 0.043 & -281 & 0.016 & -10 & 0.031 \\
\hline $\mathrm{R} 1$ & 1999 & 1204 & 0.929 & 0.014 & 0.019 & -5133 & -0.010 & 9 & 0.020 \\
\hline $\mathrm{R} 2$ & 1008 & 329 & 0.925 & 0.054 & 0.042 & -2824 & 0.033 & 13 & 0.069 \\
\hline R3 & 103 & 25 & 0.934 & 0.013 & 0.044 & -285 & 0.039 & -29 & -0.012 \\
\hline $\mathrm{R} 4$ & 695 & 20 & 0.999 & 0.010 & 0.021 & -6 & 0.001 & -6 & 0.010 \\
\hline $\mathrm{R} 5$ & 1999 & 609 & 0.990 & 0.030 & 0.120 & -3892 & 0.010 & 160 & 0.039 \\
\hline R6 & 1252 & 48 & 0.958 & 0.003 & 0.019 & -3 & -0.023 & 34 & -0.009 \\
\hline R7 & 1672 & 331 & 0.978 & 0,851 & 0.110 & -1927 & 0.008 & 145 & 0.000 \\
\hline $\mathrm{R} 8$ & 1999 & 575 & 0.976 & 0.017 & 0.044 & -3358 & 0.007 & 164 & 0.048 \\
\hline mean & & 393 & 0.961 & 0.024 & 0.052 & -2179 & 0.008 & 61 & 0.021 \\
\hline
\end{tabular}


Table 7: Alternative approaches: comparison for low level approximation

\begin{tabular}{|c|c|c|c|c|c|c|c|c|c|}
\hline & \multicolumn{3}{|c|}{ ProTraS } & \multirow{2}{*}{$\begin{array}{r}D E N D I S \\
\Delta R I\end{array}$} & \multirow{2}{*}{$\begin{array}{r}D I D E S \\
\Delta R I\end{array}$} & \multicolumn{2}{|c|}{ AdaCor } & \multicolumn{2}{|c|}{ AdaGri } \\
\hline & $\mathrm{S}$ & $\mathrm{t}(\mathrm{ms})$ & $R I$ & & & $\Delta t$ & $\Delta R I$ & $\Delta t$ & $\Delta R I$ \\
\hline $\mathrm{S} 1$ & 97 & 19 & 0.938 & 0.009 & 0.002 & -19 & 0.020 & 1 & 0.028 \\
\hline $\mathrm{S} 2$ & 116 & 23 & 0.956 & 0.023 & 0.018 & -57 & 0.036 & -4 & 0.056 \\
\hline S3 & 119 & 24 & 0.952 & 0.008 & 0.002 & -109 & 0.033 & -10 & 0.031 \\
\hline $\mathrm{S} 4$ & 261 & 68 & 0.963 & -0.005 & 0.009 & -215 & 0.032 & 22 & 0.028 \\
\hline S5 & 104 & 124 & 0.959 & 0.212 & 0.130 & -2387 & 0.106 & -158 & 0.086 \\
\hline S6 & 56 & 6 & 0.940 & 0.008 & 0.045 & 4 & 0.011 & 5 & 0.030 \\
\hline $\mathrm{S} 7$ & 96 & 15 & 0.968 & 0.020 & 0.036 & -60 & 0.034 & -11 & 0.040 \\
\hline S8 & 120 & 15 & 0.962 & 0.032 & 0.035 & -60 & 0.034 & -11 & 0.033 \\
\hline $\mathrm{S} 9$ & 155 & 14 & 0.963 & 0.015 & 0.039 & -63 & 0.015 & -14 & 0.033 \\
\hline S10 & 166 & 16 & 0.956 & 0.039 & 0.077 & -62 & 0.021 & -10 & 0.058 \\
\hline $\mathrm{S} 11$ & 15 & 15 & 0.962 & 0.007 & 0.042 & -62 & 0.020 & -11 & 0.028 \\
\hline $\mathrm{S} 12$ & 10 & 6 & 0.967 & 0.090 & 0.100 & -5 & 0.065 & 0 & 0.104 \\
\hline $\mathrm{S} 13$ & 10 & 6 & 0.988 & 0.035 & 0.022 & -29 & 0.252 & -19 & 0.306 \\
\hline S14 & 175 & 25 & 0.977 & 0.020 & 0.009 & -60 & -0.004 & -40 & 0.036 \\
\hline $\mathrm{S} 15$ & 175 & 16 & 0.991 & 0.038 & 0.016 & -109 & 0.000 & -69 & 0.007 \\
\hline $\mathrm{S} 16$ & 492 & 36 & 0.993 & 0.002 & -0.001 & -198 & 0.006 & -186 & -0.003 \\
\hline $\mathrm{S} 17$ & 152 & 20 & 0.949 & 0.012 & 0.027 & -129 & 0.009 & -25 & 0.046 \\
\hline $\mathrm{S} 18$ & 116 & 69 & 0.961 & 0.059 & 0.110 & -1639 & 0.037 & -83 & 0.031 \\
\hline S19 & 152 & 30 & 0.955 & 0.011 & 0.029 & -118 & -0.001 & -15 & 0.076 \\
\hline $\mathrm{S} 20$ & 118 & 25 & 0.965 & 0.022 & 0.093 & -63 & 0.013 & -4 & 0.036 \\
\hline $\mathrm{S} 21$ & 83 & 31 & 0.967 & -0.006 & 0.098 & -240 & 0.002 & -26 & 0.001 \\
\hline mean & & 28 & 0.963 & 0.031 & 0.045 & -270 & 0.035 & -32 & 0.052 \\
\hline $\mathrm{R} 1$ & 1999 & 1198 & 0.931 & 0.027 & 0.157 & -4900 & 0.002 & 267 & 0.025 \\
\hline $\mathrm{R} 2$ & 321 & 179 & 0.882 & 0.148 & 0.130 & -2477 & 0.020 & -125 & 0.104 \\
\hline R3 & 56 & 20 & 0.891 & -0.023 & 0.007 & -281 & -0.018 & -34 & 0.005 \\
\hline $\mathrm{R} 4$ & 508 & 13 & 0.997 & 0.005 & 0.022 & -9 & 0.004 & -13 & 0.008 \\
\hline $\mathrm{R} 5$ & 809 & 294 & 0.978 & 0.013 & 0.109 & -2878 & 0.019 & -177 & 0.042 \\
\hline $\mathrm{R} 6$ & 488 & 17 & 0.854 & 0.087 & 0.099 & -14 & 0.002 & 5 & 0.010 \\
\hline $\mathrm{R} 7$ & 396 & 116 & 0.964 & 0,804 & 0.097 & -1422 & 0.003 & -110 & 0.029 \\
\hline $\mathrm{R} 8$ & 795 & 286 & 0.971 & 0.042 & 0.083 & -2515 & 0.007 & -162 & 0.037 \\
\hline mean & & 265 & 0.935 & 0.038 & 0.088 & -1812 & 0.005 & -74 & 0.033 \\
\hline
\end{tabular}


to the high approximation level.

The similarity of the results yielded by ProTraS and AdaCor is not surprising: they share the idea of tracking dense areas without forgetting the others. In the AdaCor approach, dense areas are first covered by a biased random sampling, then the initial patterns represented by this sample are no longer considered giving more opportunity for less dense areas to be represented. ProTraS is clearly faster than $A d a C o r$ and this is more visible for large data sets $(S 5, S 17$ and most of the real world ones). The algorithm complexities are similar but the number of distance calculations is reduced in ProTraS thanks to an internal optimization.

In contrast, AdaGri may be faster than ProTraS but is outperformed by the latter. Although adaptive, the grid bins are likely to mix outliers or noisy data with more representative ones. Yet, the same bias is applied to the whole cell.

DIDES showed the worst performance for the same sample size. This was expected as the main goal of this algorithm is to ensure space coverage. DENDIS, whose aim is density representation, gave intermediate results.

To summarize, ProTraS proved to give similar, even better, results compared with AdaCor while being as fast as AdaGri, and without any post-processing step, in contrast with DENDIS or DIDES. The main characteristics of the compared algorithms are given in Table 8 
Table 8: Comparison of the different algorithms

\begin{tabular}{|c|c|c|c|c|c|}
\hline & Input params & $\begin{array}{l}\text { Internal } \\
\text { params }\end{array}$ & $\begin{array}{l}\text { Stopping } \\
\text { criterion }\end{array}$ & Cons & Pros \\
\hline DIDES & Granularity & Yes & Distance & $\begin{array}{c}\text { Post-processing } \\
\text { on density }\end{array}$ & $\begin{array}{c}\text { Space } \\
\text { coverage }\end{array}$ \\
\hline DENDIS & Granularity & Yes & Density & $\begin{array}{c}\text { Post-processing } \\
\text { on distance }\end{array}$ & $\begin{array}{c}\text { Density } \\
\text { representation }\end{array}$ \\
\hline AdaCor & $\begin{array}{c}\beta, \varepsilon \text { or } \\
\text { sample size }\end{array}$ & Yes & $\begin{array}{c}\varepsilon \text { or } \\
\text { sample size }\end{array}$ & Slow & Hybrid \\
\hline AdaGri & $\begin{array}{c}\alpha, N_{c u t}, \\
\operatorname{Max}_{b}, O c c_{m}\end{array}$ & No & $\begin{array}{c}\text { Based on } \\
\text { input params }\end{array}$ & $\begin{array}{l}\text { Tuning may } \\
\text { be difficult }\end{array}$ & Fast \\
\hline ProTraS & $\varepsilon$ & No & $\varepsilon$ & & Fast, $\varepsilon$ \\
\hline
\end{tabular}

\subsection{Wilcoxon test}

To assess how significant the differences between ProTraS and the four studied algorithms are, a Wilcoxon signed-rank test was performed on the mean $R I$ values. The 29 synthetic and real world data sets were considered. The test was based upon the sign of the difference of the observed values, and the $R$ Project ${ }^{6}$ implementation was used. The results are given in Table 9

Table 9: Wilcoxon signed-rank test results

\begin{tabular}{c|c|rrrrr}
\hline Approx level & & ProTraS & DENDIS & DIDES & AdaCor & AdaGri \\
\hline high & mean & 0.974 & 0.955 & 0.929 & 0.960 & 0.946 \\
& p-value & & 0.00242 & 0.00008 & 0.00421 & 0.00005 \\
\hline \multirow{2}{*}{ low } & mean & 0.955 & 0.923 & 0.899 & 0.929 & 0.909 \\
& $p$-value & & 0.00632 & 0.00001 & 0.00980 & 0.00015 \\
\hline
\end{tabular}

For the two studied approximation levels the $R I$ means, over the 29 data sets are reported for ProTraS and the alternative approach considered. The p-value

\footnotetext{
${ }^{6}$ https://r-project.org, wilcox.test function with paired=TRUE.
} 
is the probability of observing the computed statistic (not shown), given the experimental conditions, under the null hypothesis, meaning that both $R I$ values come from a unique population, i.e. there is no difference between ProTraS and its competitor.

The Wilcoxon test clearly supports the rejection of the null hypothesis in all cases: the $p$-value are all below $1 \%$. As stated before, the closest results are obtained with AdaCor for the low approximation level.

\section{Conclusion}

The new sampling algorithm proposed in this paper achieves an iterative partitioning of the input space based on distance and density considerations. At each step a new item is added to the sample set. It is chosen as the farthest from the already selected one in a given group. ProTraS is a fft-based algorithm.

It is shown in the present work that such fft algorithms yield $(k, \varepsilon)$-coresets. For a given group, $i$, the approximation is proportional to the sampling cost, i.e. the product of the maximum within group distance, $d_{i}$, and the group cardinality, $w_{i}$. As these data are updated at each step of the algorithm, an upper estimate of the sampling cost is available without any additional computational effort.

ProTraS, in contrast to previous fft algorithms such as DIDES or DENDIS, aims to design a coreset. Hence, the sampling cost is given the central role. First, it is the unique and meaningful parameter. Second, it also serves as the stopping criterion: the algorithm stops when the desired approximation level is reached. No hidden parameters or procedures are required. Finally, it is also used to guide the sampling process: at each step the new representative is added to the group with the highest probability of cost reduction. This value is the combination of two basic probabilities: one according to the within group distance, the other according to the group cardinality. The semantics are the same: the higher the distance or the cardinality, the higher the probability. The probabilistic approach in ProTraS achieves a trade-off between space coverage 
and density representation.

The tests carried out on a synthetic data set already used in Kärkkäinen \& Fränti, 2002) show that ProTraS shares some properties with the previous two fft algorithms. It is robust to noise, the sample size mainly depends on the data structure instead of on the data size and it is time optimized. As the first steps of the algorithm are the most time consuming, a grid approach could really impact the process time.

Numerical experiments using 29 data sets, both synthetic and real world, show that the sample yielded by ProTraS is representative of the initial set. For two famous clustering algorithms, k-means and DBSCAN, the partitions computed from the whole and the sample are similar. The time ratio, i.e. sampling time plus time to cluster the sample over time to cluster the whole data, proved extremely low for a smart, but costly, algorithm like $D B S C A N$, enhancing the usefulness of the sampling approach. Using the $k$-means, the interest of ProTraS is restricted to large and/or structured data sets. The sensitivity to the unique parameter showed that for a large range of distortion costs, the sample is able to accurately represent the whole according to the Rand Index, even with a reduced size. A comparison with competitors selected from previous studies showed that ProTraS yielded the best $R I$ mean on average over all the data sets for the studied configurations. A Wilcoxon signed-rank test clearly supported the alternative hypothesis, i.e. the two $R I$ series come from two distinct distributions, the ProTraS and the competitor ones, in all the configurations with a p-value below $1 \%$.

The proposal could be improved to deal with a huge number of observations. Several ways could be explored or combined. To begin with, it is possible to speed up the first steps of the algorithm, using a grid. This is likely to impact the running time as the first steps are the most expensive ones. Other optimization techniques can also be envisaged such as a kd-tree implementation or a neighborhood graph.

A stratification strategy could be developed He et al. (2011): divide the strata, run the algorithm on each subset and combine the separate samples to 
yield the final result. As there is no post-processing stage, a streaming version Guha \& Mishra (2016) can also be imagined.

The main challenge is to deal with high dimensional data, to understand and visualize these data. The most common approaches propose a space reduction, using a feature selection or a space transform process, before sampling. These two objectives can be combined in a single optimization problem to design a system that performs a dual selection of features and patterns according to a given criterion. Some attempts are based on evolutionary algorithms Ros \& Guillaume (2007). The proposal could be adapted to be included in such a system.

\section{References}

Agarwal, P. K., Har-Peled, S., \& Varadarajan, K. R. (2004). Approximating extent measures of points. J. ACM , 51,606-635. doi:http://doi.acm.org/ 10.1145/1008731.1008736.

Alcalá, J., Fernández, A., Luengo, J., Derrac, J., García, S., Sánchez, L., \& Herrera, F. (2010). Keel data-mining software tool: Data set repository, integration of algorithms and experimental analysis framework. Journal of Multiple-Valued Logic and Soft Computing, 17, 255-287.

Arthur, D., \& Vassilvitskii, S. (2007). k-means++: The advantages of careful seeding. In Proceedings of the eighteenth annual ACM-SIAM symposium on Discrete algorithms (pp. 1027-1035). Society for Industrial and Applied Mathematics.

Celebi, M. E., Kingravi, H. A., \& Vela, P. A. (2013). A comparative study of efficient initialization methods for the k-means clustering algorithm. Expert Systems with Applications, 40, 200-210.

Feldman, D., Faulkner, M., \& Krause, A. (2011). Scalable training of mixture models via coresets. In Advances in Neural Information Processing Systems (pp. 2142-2150). 
Fränti, P., \& Virmajoki, O. (2006). Iterative shrinking method for clustering problems. Pattern Recognition, 39, 761-765.

Fu, L., \& Medico, E. (2007). Flame, a novel fuzzy clustering method for the analysis of dna microarray data. BMC bioinformatics, 8,3 .

Gonzalez, T. F. (1985). Clustering to minimize the maximum intercluster distance. Theoretical Computer Science, 38, 293 - 306. URL: http://www. sciencedirect.com/science/article/pii/0304397585902245, doi/http: //dx.doi.org/10.1016/0304-3975(85)90224-5

Guha, S., \& Mishra, N. (2016). Clustering data streams. In Data Stream Management (pp. 169-187). Springer.

Har-Peled, S., \& Mazumdar, S. (2004). On coresets for k-means and k-median clustering. In Proceedings of the Thirty-sixth Annual ACM Symposium on Theory of Computing STOC '04 (pp. 291-300). New York, NY, USA: ACM. doi $10.1145 / 1007352.1007400$

Hartigan, J. A. (1975). Clustering Algorithms. Wiley.

Hartigan, J. A., \& Wong, M. (1979). A k-means clustering algorithm. Applied Statistics, 28, 100-108.

He, Y., Tan, H., Luo, W., Mao, H., Ma, D., Feng, S., \& Fan, J. (2011). Mrdbscan: an efficient parallel density-based clustering algorithm using mapreduce. In Parallel and Distributed Systems (ICPADS), 2011 IEEE 17th International Conference on (pp. 473-480). IEEE.

Ilango, M. R., \& Mohan, V. (2010). A survey of grid based clustering algorithms. International Journal of Engineering Science and Technology, 2, 3441-3446.

Jain, A., \& Law, M. (2005). Data Clustering: A User's Dilemma. In Proceedings of the First international conference on Pattern Recognition and Machine Intelligence (pp. 1-10). 
Jain, A. K. (2010). Data clustering: 50 years beyond k-means. Pattern Recognition Letters, 31, 651-666.

Kärkkäinen, I., \& Fränti, P. (2002). Dynamic local search algorithm for the clustering problem. Technical Report A-2002-6 Department of Computer Science, University of Joensuu Joensuu, Finland.

Karypis, G., Han, E.-H., \& Kumar, V. (1999). Chameleon: Hierarchical clustering using dynamic modeling. Computer, 32, 68-75.

Kollios, G., Gunopulos, D., Koudas, N., \& Berchtold, S. (2003). Efficient biased sampling for approximate clustering and outlier detection in large data sets. IEEE Transactions on Knowledge and Data Engineering, 15, 1170-1187.

Lin, Y., Cunningham, G. A., \& Coggeshall, S. V. (1997). Using fuzzy partitions to create fuzzy systems from input-output data and set the initial weights in a fuzzy neural network. IEEE Transactions on Fuzzy Systems, 5, 614-621.

Linde, Y., Buzo, A., \& Gray, R. (1980). An algorithm for vector quantizer design. IEEE Transactions on Communications, 28, 84-95. doi 10.1109/ TCOM.1980.1094577.

Ling, R. F. (1981). Cluster analysis algorithms for data reduction and classification of objects. Technometrics, 23, 417-418.

Lloyd, S. P. . (1982). Least squares quantization in pcm. IEEE Trans. Inf. Theor., 28, 129-137.

Lv, Y., Ma, T., Tang, M., Cao, J., Tian, Y., Al-Dhelaan, A., \& Al-Rodhaan, M. (2015). An efficient and scalable density-based clustering algorithm for datasets with complex structures. Neurocomputing, In Press.

Ma, X., Pan, Z., Li, Y., \& Fang, J. (2015). High-quality initial codebook design method of vector quantisation using grouping strategy. IET Image Processing, 9, 986-992. 
Machová, K., Puszta, M., Barčák, F., \& Bednár, P. (2006). A comparison of the bagging and the boosting methods using the decision trees classifiers. Computer Science and Information Systems, 3, 57-72.

Macqueen, J. (1967). Some methods for classification and analysis of multivariate observations. In In 5-th Berkeley Symposium on Mathematical Statistics and Probability (pp. 281-297).

Palmer, C. R., \& Faloutsos, C. (2000). Density biased sampling: An improved method for data mining and clustering. In ACM SIGMOD Intl. Conference on Management of Data (pp. 82-92). Dallas.

Pintore, M., Audouze, K., Ros, F., \& Chretien, J. R. (2002). Adaptive fuzzy partition in database mining: application to olfaction. Data Science Journal, $1,99-110$.

Rand, W. M. (1971). Objective criteria for the evaluation of clustering methods. Journal of the American Statistical Association, 66, 846850.

Ros, F., \& Guillaume, S. (2007). An efficient nearest neighbor classifier. In Hybrid Evolutionary Systems (pp. 131-150). Hybrid Evolutionary Systems, Studies in Computational Intelligence, Vol 75, Springer.

Ros, F., \& Guillaume, S. (2016a). Dendis: A new density-based sampling for clustering algorithm. Expert Systems with Applications, 56, 349-359. doi 10. 1016/j.eswa.2016.03.008.

Ros, F., \& Guillaume, S. (2016b). Dides: a fast and effective sampling for clustering algorithm. Knowledge and Information Systems, (p. In press). doi: $10.1007 / \mathrm{s} 10115-016-0946-8$.

Rosenkrantz, D. J., Stearns, R. E., \& Lewis, P. M., II (1977). An analysis of several heuristics for the traveling salesman problem. SIAM journal on computing, 6, 563-581. 
Sarma, T. H., Viswanath, P., \& Reddy, B. E. (2013). Speeding-up the kernel k-means clustering method: A prototype based hybrid approach. Pattern Recognition Letters, 34, 564-573.

Thompson, S. K. (2012). Sampling. (3rd ed.). Wiley.

Tran, T. N., Drab, K., \& Daszykowski, M. (2013). Revised dbscan algorithm to cluster data with dense adjacent clusters. Chemometrics and Intelligent Laboratory Systems, 120, 92-96.

Viswanath, P., Sarma, T., \& Reddy, B. (2013). A hybrid approach to speed-up the k-means clustering method. International Journal of Machine Learning and Cybernetics, 4, 107-117.

Wang, L., Leckie, C., Kotagiri, R., \& Bezdek, J. (2011). Approximate pairwise clustering for large data sets via sampling plus extension. Pattern Recognition, 44, 222-235.

Xiang, Z. (1997). Color image quantization by minimizing the maximum intercluster distance. ACM Trans. Graph., 16, 260-276. URL: http://doi.acm. org/10.1145/256157.256159, doi $10.1145 / 256157.256159$

Yager, R. R., \& Filev, D. P. (1994). Generation of fuzzy rules by mountain clustering. Journal of Intelligent and Fuzzy Systems, 2, 209-219.

Zahra, S., Ghazanfar, M. A., Khalid, A., Azam, M. A., Naeem, U., \& PrugelBennett, A. (2015). Novel centroid selection approaches for kmeans-clustering based recommender systems. Information Sciences, .

Zhang, T., Ramakrishnan, R., \& Livny, M. (1997). Birch: A new data clustering algorithm and its applications. Data Mining and Knowledge Discovery, 1, $141-182$.

Zhong, C., Malinen, M., Miao, D., \& Fränti, P. (2015). A fast minimum spanning tree algorithm based on k-means. Information Sciences, 295, 1-17. 
Author-produced version of the article published in Expert Systems with Applications, 2018, №105, p. 65-76.

The original publication is available at https://www.sciencedirect.com

Doi: 10.1016/j.eswa.2018.03.052

Zhou, S., Zhou, A., Jin, W., Fan, Y., \& Qian, W. (2000). Fdbscan: a fast dbscan algorithm. Ruan Jian Xue Bao, 11, 735-744. 